\title{
Hyperentanglement-assisted Bell-state analysis
}

\author{
S. P. Walborn $*$ S. Pádua, and C. H. Monken \\ Universidade Federal de Minas Gerais, Caixa Postal 702, Belo Horizonte, MG 30123-970, Brazil
}

(Dated: November 11, 2018)

\begin{abstract}
We propose a simple scheme for complete Bell-state measurement of photons using hyperentangled states - entangled in multiple degrees of freedom. In addition to hyperentanglement, our scheme requires only linear optics and single photon detectors, and is realizable with current technology. At the cost of additional classical communication, our Bell-state measurement can be implemented nonlocally. We discuss the possible application of these results to quantum dense coding and quantum teleportation.

PACS numbers: 03.67.-a,03.67.Hk,42.50.-p
\end{abstract}

\section{INTRODUCTION}

Bell-state measurement (BSM) - distinguishing between the four maximally-entangled Bell states - is required in many quantum information schemes, including quantum dense coding [1, 2], quantum teleportation [3, 4, 5] and entanglement swapping [3, 6, 7]. However, it has been proven that a complete BSM (distinguishing between the four states with $100 \%$ efficiency) is impossible using only linear operations and classical communication [8, 9, 10, 11]. In fact, Ghosh et. al. [11] have proven that, if only a single copy is provided, the best one can do is discriminate between two Bell states. Calsamiglia and Lütkenhaus 10 have shown that the maximum efficiency for a linear Bell-state analyzer is $50 \%$.

A favorable characteristic of the photon as a carrier of quantum information is the relative ease with which entangled photons can be created and transported. Twophoton Bell states are easily generated using spontaneous parametric down-conversion (SPDC) in one of several degrees of freedom 12, 13, 14, 15. There are several methods for optical Bell-state measurement that allow one to distinguish two [2, 16, 17, 18, 19] of the four Bell-states states (resulting in 3 classes of states). All of these methods use local or non-local two-photon interference effects at beam splitters. For example, Mattle et. al. 2] have performed an experimental demonstration of dense coding, in which the Bell-states were created in the polarization degrees of freedom of the two photons. When the photons meet at a common beam splitter, the overall bosonic symmetry of the two-photon state requires that photons in the antisymmetric singlet state exit in different output ports, while the symmetric triplet states end up in the same output port [20]. Polarization analyzers are then used to further discriminate among the triplet states. Weinfurter has proposed a BSM method using momentum-entangled photons that allows one to distinguish all four Bell-states with 25\% efficiency [16]. It is possible to distinguish among the four Bell states using nonlinear optical processes [21, 22], however, with

*swalborn@fisica.ufmg.br present technology these methods suffer from low efficiency. There have also been several proposals using twophoton absorption [23, 24].

In recent years, some attention has been paid to Bellstate analysis using hyperentangled states 19, 25]. Utilizing entanglement in additional auxiliary degrees of freedom, it is possible to perform a complete BSM. Due to the enlarged Hilbert space, this type of complete BSM is not restricted to the efficiency limits presented in [8, 9, 10, 11]. Kwiat and Weinfurter 25] have proposed a scheme using photons entangled in polarization and momentum (spatial mode). Their method, which relies on linear optics and two-photon interference effects, requires detectors that distinguish between one- and two-photon detection events. We showed that this requirement on the detectors could be removed if the hyperentangled photons were created by SPDC using a Hermite-Gaussian pump beam [19]. The Hermite-Gaussian beam used is an odd function of the horizontal transverse spatial coordinate, which inverts the two-photon interference behavior [26], allowing for identification of all four Bell-states in coincidence detections.

Here we present a new method for complete Bell-state analysis using hyperentangled states. This scheme differs from others in that it $(i)$ does not rely on two-photon interference, (ii) does not require detectors sensitive to photon number, and ( $i i i$ ) can be implemented non-locally with 2 bits of additional classical communication. In section [I] we briefly discuss the creation of hyperentangled states. We present our hyperentangled Bell-state analyzer in section III and discuss the application of these results to quantum information protocol such as dense coding and teleportation.

\section{HYPERENTANGLEMENT}

We will work with hyperentangled two-photon states of the form $|\Pi\rangle \otimes|\eta\rangle \equiv|\Pi\rangle|\eta\rangle$. Here $|\Pi\rangle$ and $|\eta\rangle$ are the four dimensional vectors representing the polarization and momentum degrees of freedom of the two photons, respectively. In the basis defined by linear horizontal $(H)$ and linear vertical $(V)$ polarization, the polarization- 


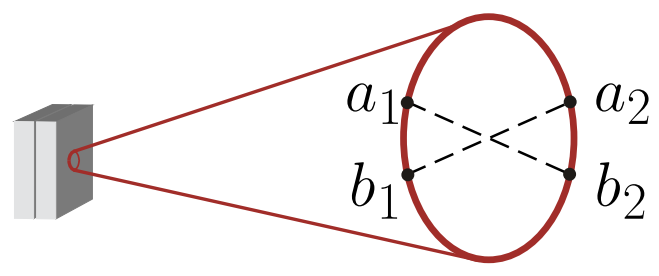

FIG. 1: Hyperentangled states can be generated using the type-I two-crystal source [15]. Selecting two sets of regions $\left(a_{1} b_{2}\right.$ and $\left.a_{2} b_{1}\right)$ gives photon pairs entangled in momentum and polarization.

entangled Bell-states are:

$$
\begin{aligned}
& \left|\Psi^{ \pm}\right\rangle=\frac{1}{\sqrt{2}}\left(|H\rangle_{1}|V\rangle_{2} \pm|V\rangle_{1}|H\rangle_{2}\right) \\
& \left|\Phi^{ \pm}\right\rangle=\frac{1}{\sqrt{2}}\left(|H\rangle_{1}|H\rangle_{2} \pm|V\rangle_{1}|V\rangle_{2}\right)
\end{aligned}
$$

where $|\sigma\rangle_{j}$ stand for the polarization state of the photon $j$. Likewise, the momentum-entangled Bell-states are:

$$
\begin{aligned}
\left|\psi^{ \pm}\right\rangle & =\frac{1}{\sqrt{2}}\left(|a\rangle_{1}|b\rangle_{2} \pm|b\rangle_{1}|a\rangle_{2}\right) \\
\left|\phi^{ \pm}\right\rangle & =\frac{1}{\sqrt{2}}\left(|a\rangle_{1}|a\rangle_{2} \pm|b\rangle_{1}|b\rangle_{2}\right)
\end{aligned}
$$

where $|a\rangle_{j}$ and $|b\rangle_{j}$ represent different spatial modes of photon $j$. We note here that polarization states have been denoted with uppercase letters and momentum states with lowercase letters.

For our hyperentangled-Bell-state analysis we will consider states of the form $|\Pi\rangle\left|\psi^{+}\right\rangle$, where $|\Pi\rangle$ is one of the polarization Bell states (10), though one could use any of the states (2) with similar results. Recently, it was shown that this type of hyperentangled two-photon state could be used to violate a generalized form of the GreenbergerHorne-Zeilinger theorem 27], and may be useful in creating decoherence-free subspaces [28]. These states can be generated by means of spontaneous parametric down-conversion (SPDC) in several ways 15, 27, 29]. For example, the type-I two-crystal source 15] emits polarization-entangled photons of the same wavelength around the rim of a cone (FIG. (1). In this source, crystal 1 emits pairs of (say) $H$-polarized photons and crystal 2 emits pairs of $V$-polarized photons in superimposed emission cones. Phase-matching conditions guarantee that photon pairs are emitted on opposite sides of the cone. If the two-crystal interaction region lies entirely within a coherence volume of the pump laser beam, a polarizationand momentum-entangled state of the form $|\Pi\rangle|\eta\rangle$ can be selected by the two sets of regions $a_{1} b_{2}$ and $a_{2} b_{1}$. One can adjust the phase so that the momentum state is $\left|\psi^{+}\right\rangle$. Half- and quarter-wave plates can be used to switch between the four polarization Bell-states [14].

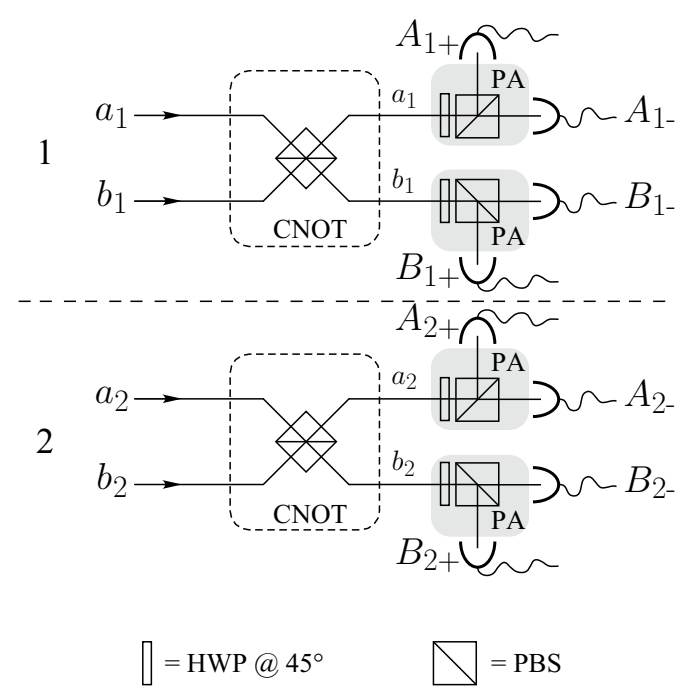

FIG. 2: The hyperentangled Bell-state analyzer. The PBS are polarizing beam splitters in the $H-V$ basis. HWP are halfwave plates. The CNOT operation uses the polarization as the control and momentum as the target. The PA are polarization analyzers in the $\pm 45^{\circ}$ basis (denoted as \pm ) consisting of a HWP and a PBS.

\section{BELL-STATE ANALYSIS}

The hyperentangled-Bell-state analyzer is shown in FIG. 2 The hyperentangled state $|\Pi\rangle\left|\psi^{+}\right\rangle$is first incident on a set of polarizing-beam splitters (PBS), which reflect $H$-polarized photons and transmit $V$-polarized photons. The PBS perform a controlled-not (CNOT) logic operation between the polarization (control) and spatial (target) degrees of freedom [30]. If the polarization is $V$ then the photon is transmitted, and switches modes. If the polarization is $H$, then the photon is reflected, and remains in the same mode. The complete PBS operation on the polarization and spatial mode of photon $j$ is

$$
\begin{aligned}
|H\rangle_{j}|a\rangle_{j} & \longrightarrow|H\rangle_{j}|a\rangle_{j} \\
|H\rangle_{j}|b\rangle_{j} & \longrightarrow|H\rangle_{j}|b\rangle_{j} \\
|V\rangle_{j}|a\rangle_{j} & \longrightarrow|V\rangle_{j}|b\rangle_{j} \\
|V\rangle_{j}|b\rangle_{j} & \longrightarrow|V\rangle_{j}|a\rangle_{j}
\end{aligned}
$$

It is straightforward to show that the four states $|\Pi\rangle\left|\psi^{+}\right\rangle$ transform as

$$
\begin{aligned}
& \left|\Psi^{ \pm}\right\rangle\left|\psi^{+}\right\rangle \longrightarrow\left|\Psi^{ \pm}\right\rangle\left|\phi^{+}\right\rangle \\
& \left|\Phi^{ \pm}\right\rangle\left|\psi^{+}\right\rangle \longrightarrow\left|\Phi^{ \pm}\right\rangle\left|\psi^{+}\right\rangle
\end{aligned}
$$

A quick look at (4) shows that the PBS's mark the momentum state by performing a polarization-controlled logic operation. The momentum state can then be used to discriminate between the $\left|\Psi^{ \pm}\right\rangle$and $\left|\Phi^{ \pm}\right\rangle$polarization states: coincidence detections in modes $a_{1} a_{2}$ or $b_{1} b_{2}$ imply states $\left|\Psi^{ \pm}\right\rangle$while coincidences in $a_{1} b_{2}$ or $b_{1} a_{2}$ imply 
TABLE I: Detector signatures for polarization Bell-states using the momentum state $\left|\psi^{+}\right\rangle$as an ancilla. "+" $\equiv+45^{\circ}$ and "-" $\equiv-45^{\circ}$.

\begin{tabular}{cc}
\hline \hline state & detector signature \\
$\left|\Psi^{+}\right\rangle$ & $A_{1+} A_{2+}$ or $B_{1+} B_{2+}$ or $A_{1-} A_{2-}$ or $B_{1-} B_{2-}$ \\
$\left|\Psi^{-}\right\rangle$ & $A_{1+} A_{2-}$ or $B_{1+} B_{2-}$ or $A_{1-} A_{2+}$ or $B_{1-} B_{2+}$ \\
$\left|\Phi^{+}\right\rangle$ & $A_{1+} B_{2+}$ or $B_{1+} A_{2+}$ or $A_{1-} B_{2-}$ or $B_{1-} A_{2-}$ \\
$\left|\Phi^{-}\right\rangle$ & $A_{1+} B_{2-}$ or $B_{1+} A_{2-}$ or $A_{1-} B_{2+}$ or $B_{1-} A_{2+}$ \\
\hline
\end{tabular}

states $\left|\Phi^{ \pm}\right\rangle$. Using additional polarization analyzers (PA in FIG. (2) orientated at $45^{\circ}$, we can discriminate between the respective \pm states. Specifically, $\left|\Psi^{+}\right\rangle$and $\left|\Phi^{+}\right\rangle$give coincidence counts at the $+45,+45$ or $-45,-45$ output ports while $\left|\Psi^{-}\right\rangle$and $\left|\Phi^{-}\right\rangle$give coincidence counts at the $-45,+45$ or $+45,-45$ output ports. A summary of the detector signatures for the polarization Bell states are shown in table

We note that all four Bell-states are recognized in the coincidence basis, with no need for detectors that are sensitive to photon number, and without the use of twophoton interference effects. Furthermore, the BSM can be performed non-locally at the cost of 2 bits of classical communication, since the physicist measuring system 1 needs to communicate one of four possible outcomes to system 2 (or vice versa).

The hyperentangled Bell-state analyzer is well within the scope of present technology, necessitating only wave plates, polarizing beam splitters and single photon detectors.

A short analysis of the stability of the scheme proposed here may be in order. In addition to mode overlap at the polarizing beam splitters, the use of the ancillary momentum state $\left|\psi^{+}\right\rangle$requires phase stability between the modes. A phase error $\alpha$ of the form

$$
\begin{aligned}
\left|\psi^{+}\right\rangle & \longrightarrow \frac{1}{\sqrt{2}}\left(|a\rangle_{1}|b\rangle_{2}+e^{i \alpha}|b\rangle_{1}|a\rangle_{2}\right) \\
& =\frac{1}{2}\left(1+e^{i \alpha}\right)\left|\psi^{+}\right\rangle+\frac{1}{2}\left(1-e^{i \alpha}\right)\left|\psi^{-}\right\rangle
\end{aligned}
$$

introduces an error in the BSM, since the CNOT operations with the ancillary momentum state $\left|\psi^{-}\right\rangle$are:

$$
\begin{aligned}
\left|\Psi^{ \pm}\right\rangle\left|\psi^{-}\right\rangle & \longrightarrow\left|\Psi^{\mp}\right\rangle\left|\phi^{-}\right\rangle, \\
\left|\Phi^{ \pm}\right\rangle\left|\psi^{-}\right\rangle & \longrightarrow\left|\Phi^{\mp}\right\rangle\left|\psi^{-}\right\rangle .
\end{aligned}
$$

The momentum states continue to show the same type of correlation, (the polarization states $\left|\Psi^{ \pm}\right\rangle$at $a_{1} a_{2}$ or $b_{1} b_{2}$, etc.), however, the polarization states have been switched. From (5), the probability to obtain the correct Bell state is thus $|1+\exp (i \alpha)|^{2} / 2$. To avoid bilateral phase errors, we could use the momentum state $\left|\psi^{-}\right\rangle$ instead of the $\left|\psi^{+}\right\rangle$as an ancilla. It has been shown that $\left|\psi^{-}\right\rangle$is insensitive to collective decoherence 28].

It is also possible to use the polarization degrees of freedom as the ancilla and encode information in the

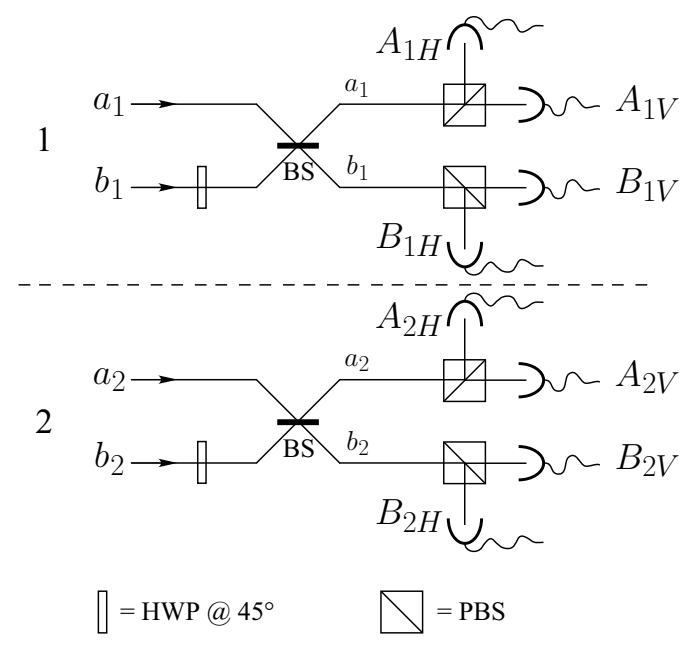

FIG. 3: The hyperentangled Bell-state analyzer for momentum Bell-states, using the polarization state as an ancilla. The CNOT operation is now performed by the half-wave plates (HWP). The BS are non-polarizing beam splitters. The PBS are polarizing beam splitters in the $H-V$ basis.

TABLE II: Detector signatures for momentum Bell-states using the polarization state $\left|\Psi^{+}\right\rangle$as an ancilla.

\begin{tabular}{cc}
\hline \hline state & detector signature \\
$\left|\psi^{+}\right\rangle$ & $A_{1 H} A_{2 H}$ or $B_{1 H} B_{2 H}$ or $A_{1 V} A_{2 V}$ or $B_{1 V} B_{2 V}$ \\
$\left|\psi^{-}\right\rangle$ & $A_{1 H} B_{2 H}$ or $B_{1 H} A_{2 H}$ or $A_{1 V} B_{2 V}$ or $B_{1 V} A_{2 V}$ \\
$\left|\phi^{+}\right\rangle$ & $A_{1 H} A_{2 V}$ or $B_{1 H} B_{2 V}$ or $A_{1 V} A_{2 H}$ or $B_{1 V} B_{2 H}$ \\
$\left|\phi^{-}\right\rangle$ & $A_{1 H} B_{2 V}$ or $B_{1 H} A_{2 V}$ or $A_{1 V} B_{2 H}$ or $B_{1 V} A_{2 H}$ \\
\hline
\end{tabular}

momentum Bell-states. A hyperentangled Bell-state analyzer for such an implementation is shown in FIG. 3 The CNOT operation is performed by half-wave plates (HWP) aligned at $45^{\circ}$ in modes $b_{1}$ and $b_{2}$. The beam splitters (BS) and polarizing beam splitters (PBS) separate the 4 hyperentangled states. The beam splitters transform the input modes as $a \longrightarrow(a+b) / \sqrt{2}$ and $b \longrightarrow(a-b) / \sqrt{2}$, which gives different correlations for the \pm states. The polarizing beam splitters separate the polarization ancilla states $\left|\Phi^{+}\right\rangle$and $\left|\Psi^{+}\right\rangle$after the CNOT operation. The detector signatures are shown in table III

We now discuss the possible application of hyperentangled Bell-state analysis to quantum information protocol. The quantum dense coding protocol [1] allows for the transmission of two bits of information in one quantum bit. Two parties, Alice and Bob, each possess one photon of an entangled Bell-state $\left(\left|\Phi^{+}\right\rangle\right.$, for example). Since the reduced density matrix for Bob's photon is $\mathbf{I} / 2$, where $\mathbf{I}$ is the $2 \times 2$ identity matrix, there is no information present in Bob's photon alone. Some time later, Alice wishes to send a 2 bit message to Bob. She switches among the four Bell-states using local operations, and then sends her photon to Bob, who performs a Bell-state measurement on the photon pair, and retrieves Alice's message. Since there was no information present in Bob's photon, 
then the 2 bits of information present was sent in Alice's photon. However, the reduced density matrix of Alice's photon is also $\mathbf{I} / 2$, so an eavesdropper could not extract any information from Alice's photon alone.

An appealing feature of the hyperentangled product states $|\Pi\rangle|\eta\rangle$ is that the two degrees of freedom can be manipulated independently. For example, one can switch among the polarization Bell states using local operations (quarter- and half-wave plates in modes $a_{j}$ and $b_{j}$ ) on the polarization state, while leaving the momentum state untouched. One could then implement a dense-coding scheme in which information is encoded in the polarization state, while the momentum state remains as an ancilla to assist in the complete Bell-state measurement. We note that this implementation requires 4 qubits (encoded in 2 photons) to transmit 2 classical bits of information and thus it may be debatable as to whether this is actually "dense" coding. However, since the density matrix of (say) photon 2 is

$$
\hat{\rho}_{2}=\operatorname{trace}_{1}\left(\hat{\rho}_{12}\right)=\frac{1}{4} \mathbf{I}_{4},
$$

where $\hat{\rho}_{12}$ is the total $16 \times 16$ density matrix of photons 1 and 2 and $\mathbf{I}_{4}$ is the $4 \times 4$ identity matrix, we can still send 2 classical bits of information in one photon (containing 2 qubits) in such a way that an eavesdropper with access to only this photon cannot extract any information.

Another use of a Bell-state measurement is in the quantum teleportation protocol [3], which can be used to swap entanglement [3, 6, 7] and perform quantum logic operations for quantum computation 31. In quantum teleportation, two spatially separate parties Alice and Bob each are in possession of one photon of an entangled pair, prepared in a Bell-state. One of them, say Alice, wants to teleport the quantum state of a third photon to Bob. Of course, Alice does not know what state she is teleporting, or else she could simply call Bob on the telephone and tell him to prepare that state. Instead, Alice performs a Bell-state measurement on her two photons. She then communicates classically to Bob, telling him the results of her BSM, at which point Bob, performs a local operation and recovers the state of the third photon, now encoded in his photon. In this way Alice can teleport a quantum state to Bob without actually knowing what state she is sending.

To date, quantum teleportation implementations 4] can be performed with a Bell-state measurement that is $50 \%$ efficient using two-photon interference, polarization analysis and single photon detectors. Given an unknown quantum state, Alice, who shares a pair of maximally entangled photons with Bob, can teleport this state to him with $50 \%$ efficiency. Suppose Alice and Bob share a pair of polarization-entangled photons in the Bell-state $\left|\Phi^{+}\right\rangle_{12}$ and Alice would like to teleport the unknown polarization state $|u\rangle_{3}$ of photon 3 . To take advantage of hyperentangled Bell-state analysis, Alice would need to entangle the momentum degree of freedom of photon 2 with that of photon 3 . To do so requires a controlled logic operation between the two photons, such as a CNOT gate. But if she could perform an efficient two-photon CNOT operation then she might as well use it to perform her BSM, which can be done with the same efficiency using a CNOT gate and a single photon Hadamard rotation 32 .

One might think that Alice could first entangle the momentum degrees of freedom of photons 2 and 3 using an inefficient CNOT gate, and discard the cases where the gate did not give the desired output (which is usually checked by measuring the ancillary modes). She could then pass photon 3 on to a friend who performs some sort of complex quantum computation using the polarization as a qubit, and then passes it back to Alice for teleportation. The complex quantum computation, which we assume to be much more difficult and time consuming than the teleportation protocol, need be performed only once, since the Bell-state measurement on photons 2 and 3 is now $100 \%$ efficient. However, since photon 3 is part of an entangled momentum state, it is now defined by 2 spatial modes. So Alice's friend is required to run the computation on both spatial modes, which in principle is the same as running the computation twice, as is required on average in the $50 \%$ efficient teleportation scheme. Thus, a teleportation protocol using Bell-state measurement of hyperentangled states of this form does not present any gain over previous methods.

\section{CONCLUSION}

We have shown a simple method for complete Bellstate analysis using hyperentangled photons. Our scheme requires only linear optics and single photon detectors, can be implemented non-locally with 2 bits of classical communication, and is well within the bounds of current technology. We have briefly discussed the application of these results to the quantum teleportation protocol. Given that our scheme requires photons entangled in multiple degrees of freedom, it does not provide any increase in efficiency to Bell-state measurements for quantum teleportation. However, our method can be applied directly to implementations of quantum dense coding, resulting in the secure transmission of 2 bits of classical information in one photon (containing 2 qubits).

\section{Acknowledgments}

The authors acknowledge financial support from the Brazilian funding agencies CNPq, CAPES and Instituto do Milênio de Informação Quântica. 
[1] C. H. Bennett and S. J. Wiesner, Phys. Rev. Lett. 69, 2881 (1992).

[2] K. Mattle, H. Weinfurter, P. G. Kwiat, and A. Zeilinger, Phys. Rev. Lett. 76, 4656 (1996).

[3] C. H. Bennett, G. Brassard, C. Crépeau, R. Jozsa, A. Peres, and W. K. Wootters, Phys. Rev. Lett. 70, 1895 (1993).

[4] D. Bouwmeester, J. Pan, K. Mattle, M. Eibl, H. Weinfurter, and A. Zeilinger, Nature 390, 575 (1997).

[5] D. Boschi, S. Branca, F. De Martini, L. Hardy, and S. Popescu, Phys. Rev. Lett. 80, 1121 (1998).

[6] J.-W. Pan, D. Bouwmeester, H. Weinfurter, and A. Zeilinger, Phys.Rev. Lett. 80, 3891 (1998).

[7] T. Jennewein, G. Weihs, J.-W. Pan, and A. Zeilinger, Phys. Rev. Lett. 88, 017903 (2002).

[8] L. Vaidman and N. Yoran, Phys. Rev. A 59, 116 (1999).

[9] N. Lütkenhaus, J. Calsamiglia, and K.-A. Suominen, Phys. Rev. A 59, 3295 (1999).

[10] J. Calsamiglia and N. Lütkenhaus, Appl. Phys. B 72, 67 (2001).

[11] S. Ghosh, G. Kar, A. Roy, A. Sen(De), and U. Sen, Phys. Rev. Lett. 87, 277902 (2001).

[12] J. G. Rarity and P. R. Tapster, Phys. Rev. Lett. 64, 2495 (1990).

[13] P. R. Tapster, J. G. Rarity, and P. C. M. Owens, Phys. Rev. Lett. 73, 1923 (1994).

[14] P. G. Kwiat, K. Mattle, H. Weinfurter, A. Zeilinger, A. V. Sergienko, and Y. Shih, Phys. Rev. Lett. 75, 4337 (1995).

[15] P. G. Kwiat, E. Waks, A. G. White, I. Appelbaum, and P. H. Eberhard, Phys. Rev. A. 60, R773 (1999).

[16] H. Weinfurter, Europhys. Lett. 25, 559 (1994).
[17] S. L. Braunstein and A. Mann, Phys. Rev. A. 51, R1727 (1995).

[18] M. Michler, K. Mattle, H. Weinfurter, and A. Zeilinger, Phys. Rev. A. 53, R1209 (1996).

[19] S. P. Walborn, A. N. de Oliveira, S. Pádua, and C. H. Monken, Europhys. Lett 62, 161 (2003).

[20] A. Zeilinger, H. J. Bernstein, and M. A. Horne, J. Mod. Optics 41, 2375 (1994).

[21] M. G. A. Paris, M. B. Plenio, S. Bose, D. Jonathan, and G. M. D'Ariano, Phys. Lett. A 273, 153 (2000).

[22] Y.-H. Kim, S. P. Kulik, and Y. Shih, Phys. Rev. Lett. 86, 1370 (2001).

[23] M. O. Scully, B.-G. Englert, and C. J. Bednar, Phys. Rev. Lett. 83, 4433 (1999).

[24] E. Del Re, B. Crosignani, and P. DiPorto, Phys. Rev. Lett. 84, 2989 (2000).

[25] P. G. Kwiat and H. Weinfurter, Phys. Rev. A 58, R2623 (1998).

[26] S. P. Walborn, A. N. de Oliveira, S. Pádua, and C. H. Monken, Phys. Rev. Lett 90, 143601 (2003).

[27] Z.-B. Chen, J.-W. Pan, Y.-D. Zhang, C. Brukner, and A. Zeilinger, Phys. Rev. Lett. 90, 160408 (2003).

[28] P. G. Kwiat, A. Berglund, J. Altepeter, and A. White, Science 290, 498 (2000).

[29] P. G. Kwiat, J. Mod. Optics 44, 2173 (1997).

[30] C. Simon and J.-W. Pan, Phys. Rev. Lett. 89, 257901 (2002).

[31] D. Gottesman and I. L. Chuang, Nature 402, 390 (1999).

[32] M. Nielsen and I. Chuang, Quantum Computation and Quantum Information (Cambridge, Cambridge, 2000). 